
25 Research Square
Preprints are preliminary reports that have not undergone peer review.
They should not be considered conclusive, used to inform clinical practice, or referenced by the media as validated information.

\title{
To Study the Relationship between the Expression of MiR-21, MiR-155, Mir- 182 , and Mir-437 and Inflammatory Factors in the Cerebrospinal Fluid of Patients with Multiple Sclerosis
}

\author{
Vahidreza Karamad \\ EGE university \\ Fatma Sogutlu \\ ege university \\ Samane Abbasi \\ Guilan University of Medical Sciences \\ behrouz shademan \\ Ege University: Ege Universitesi \\ Delara Laghousi \\ Tabriz Medical University: Tabriz University of Medical Sciences \\ Mohammad Nouri \\ Tabriz University of Medical Sciences \\ Mehdi Hassanpour \\ Tabriz Medical University: Tabriz University of Medical Sciences \\ Alireza Nourazarian ( $\nabla$ alinour65@gmail.com ) \\ Tabriz University of Medical Sciences https://orcid.org/0000-0003-2082-1335
}

Research

Keywords: Multiple Sclerosis (MS), MicroRNAs, Inflammatory markers, Receiver Operating Characteristics (ROC).

Posted Date: November 4th, 2021

DOI: https://doi.org/10.21203/rs.3.rs-960400/v1

License: (1) This work is licensed under a Creative Commons Attribution 4.0 International License. Read Full License 


\section{Abstract}

Background: MicroRNAs (miRNAs) affect the differentiation and function of inflammatory cells. In addition, miRNAs can regulate the expression of proinflammatory genes in neuron cells. Therefore, the relationship between the expression of miRNAs and inflammatory markers in the cerebrospinal fluid of individuals with multiple sclerosis was investigated.

Methods: RT-PCR determined the expression levels of MiR-21, MiR-155, Mir-182, and Mir-437 in CSF samples from patients with multiple sclerosis (MS) and the control group. The levels of the inflammatory cytokines IL $-1 \beta$, IL -6, and TNF- $\alpha$ in CSF were measured by ELISA. The level of high-sensitivity C-reactive protein (hs-CRP) was measured by the quantitative turbidometric technique.

Results: The expression of miRNAs and inflammatory factors were higher in the CSF of patients with MS than in the control group, and this difference was significant $(P<0.05)$. The results of Receiver Operating Characteristics $(R O C)$ analysis show that the area under the curve was obtained for miRNA-21 $(A U C=$ $0.97, \mathrm{P}<0.0001$ ), miRNA-182 (AUC $=0.97, \mathrm{P}<0.0001$ ), and miRNA-155 (AUC $=0.96, \mathrm{P}<0.0001$ ). The highest rate of correct diagnoses of MS was associated with miRNA-155 in CSF. The relationship between inflammatory cytokines and miRNA-21, miRNA-155, and miRNA-182 was statistically significant, but there was an indirect and moderate correlation between miRNA-437 and hs-CRP, which were also statistically significant.

Conclusion: Our results showed that the CSF levels of IL $-1 \beta$, IL -6 , TNF- $a$, and hs-CRP, and selected miRNAs, serve as biomarkers of central nervous system (CNS) inflammation and neurodegenerative processes in patients with MS.

\section{Background}

MS is one of the autoimmune diseases in which a lack of immune defense is one of the main problems in the human CNS. Despite the lack of understanding of the exact involvement of the immune system in disease, there are still many critical elements $[1,2]$. These include the immunological inability to distinguish self from non-self, persistent CNS inflammation, and sudden changes in adaptive immunity. Gray matter diseases contribute to developing disability in MS. Demyelination, neuronal loss/atrophy, and decreased synapse or glial density contribute to their development [3]. Microglia and astroglia are activated by invading T cells and contribute to these synaptic and neuronal changes [4].

Cerebrospinal fluid (CSF) is necessary to maintain brain tissue's chemical stability and eliminate metabolic toxins and waste products. Therefore, the molecular composition of CSF is like that of the brain. CSF analysis is a potential source of novel biomarkers for neurodegenerative diseases. It allows direct assessment of specific inflammatory processes in the CNS and detecting immunological pattern changes associated with disease progression [5]. Additionally, inflammatory markers such as interleukin-1, interleukin-6, TNF- and CRP have been detected in the CSF of individuals with MS [6, 7]. Cytokines are necessary for activating the immune system and are involved in the development and maintenance of an inflammatory milieu. Cytokine profiles in the CSF of patients with MS are limited and inconsistent [8]. Numerous studies indicate that sera from MS patients have elevated levels of IL-6. However, studies suggest no quantitative change compared to control groups or only minor signs [9-11]. During relapses and in association with existing neurological impairment, the serum of MS patients with relapsing-remitting disease has elevated IL-6 [12, 13].

CRP is one of many acute-phase reactants released by liver tissue in response to injury, infection, or inflammation when various stimuli activate specific receptors for the cytokins interleukin-1 and interleukin-6 $[14,15]$. In many studies, CRP levels were determined in the blood of people with different clinical subtypes of MS that had the typical and characteristic pathophysiology at the time of sampling; for example, plasma and serum levels of the protein were measured in patients with clinically undifferentiated MS and during relapsing-remitting disease and were within the reference range during remission and the progressive phase of the disease [16,17]. Elevated blood protein levels have been found in individuals with relapsing-remitting MS during clinical remission, primary and secondary progressive states, and even in pregnant women with MS $[18,19]$. Therefore, further studies are needed to determine the cytokine profile in CSF and its role in the pathogenesis of MS. MicroRNAs (miRNAs) are short non-coding RNAs that are 18-25 nucleotides in length. They are synthesized as primary miRNAs from DNA sequences and then further processed into precursor and mature miRNAs. MiRNAs with good or near-perfect complementary binding to their target mRNAs negatively affect gene expression by promoting mRNA degradation or decreasing mRNA translation [20]. All body fluids and tissues and most cell types contain microRNAs [21, 22].

The molecular mechanisms behind inflammation-induced synaptopathy are largely unclear. MiRNAs exert control over various physiological and pathological processes by post-transcriptionally repressing messenger RNAs (mRNAs) and are good candidates for inflammatory synaptic damage [23]: MicroRNAs influence inflammatory cell differentiation and function. MiRNA-182 is a transcription factor that regulates FOX01 expression in T cells and affects cell differentiation and activation. Additionally, miRNA -21 promotes T cell progression to Th1, IFN production, and Foxp3 expression. In astrocyte cells, miRNA155 controls the expression of pro-inflammatory genes [24]. MiRNA -155 expression was detected in reactive astrocytes in MS lesions [25]. Suppression of miRNA -155 increases the pro-inflammatory cytokine IL-1 [26].

Considering the crucial importance of MS and the lack of effective treatment, correct diagnosis, and prognosis, it is essential to investigate and discover the pathogenic molecular pathways in MS. This study aimed to investigate the relationship between the expression of miRNA-21, miRNA-155, miRNA-182, and miRNA-437 and inflammatory markers in the cerebrospinal fluid of people with multiple sclerosis.

\section{Methods}

\section{Sample selection}


This research is a case-control study approved by the Tabriz University of Medical Sciences' Local Ethics Committee IR. TBZMED. REC.1399. 765.Each participant completed a written informed consent form, indicating that they were aware of the risks. Fifty subjects were selected for this study from Razi Hospital (Tabriz, Iran) and specialized and subspecialized clinics at Tabriz University of Medical Sciences under the supervision of a neurologist. The control group was selected from the subjects whose biochemical and CSF Analysis with MRI results was normal. MS patients were diagnosed by an experienced neurologist using standard serum and CSF biomarkers. The McDonald criteria were used to interpret the MRI findings [27]. The study focused on individuals with relapsing-remitting multiple sclerosis (RRMS). Twenty-five patients ( 12 males and 13 females) were enrolled in the case group, while 25 subjects ( 10 males and 15 females) were enrolled in the control group. Questionnaires were used to collect demographic data such as age, sex, age of onset, disease duration, and therapy. (Table 1). As a control group, we used the remaining normal CSF from healthy subjects with no history of neurodegenerative disease, specifically MS, with normal biochemical and MRI findings. Confounding factors were excluded much, such as chronic diseases like liver, kidney and diabetes, infectious diseases, cancer, allergies, diseases based on immunity, use of immunosuppressive drugs (corticosteroids), use of dietary supplements and smoking. It contains vitamins C, E, selenium, beta-carotene, and Q-10 during the last 3 months and causes blood diseases and heart problems during strenuous physical activity.

\section{Sampling}

According to established CSF collection protocols, immediately after lumbar puncture, to extract cell-free CSF, CSF was centrifuged at $400 \mathrm{~g}$ for 15 min. CSF and RNA were extracted from cell-free cultures and stored at $80^{\circ} \mathrm{C}$ until all RNA was extracted [27].

\section{Analysis of circulating miRNAs}

\section{Circulating RNA extraction and purification}

Total RNA of CSF was isolated according to the manufacturer's instructions using the mirVana PARIS Isolation Kit (Applied Biosystems). In the procedure, a denaturing solution was diluted 2 to one with $300 \mu \mathrm{L}$ CSF. An equal amount of acid phenol and chloroform was then mixed. The aqueous phase was collected after centrifugation and combined with $100 \%$ ethanol on a filter cartridge. It was then washed thrice with $40 \mu \mathrm{L}$ of nuclease-free water.

\section{Circulating miRNA RT and pre-amplification}

In this study, reverse transcription was performed with a constant volume of $3 \mu \mathrm{L}$ of total pure DNA. Following the published results, seven hundred fifty-four miRNAs were isolated from CSF samples and prepared using the TaqMan miRNA Reverse Transcription Kit from Applied Biosystems and Multiplex RT assays (Human Pool Sets A and B). We used TaqMan PreAmp Master Mix and Megaplex PreAmp primers from Applied Biosystems (Human Pool Sets A and B) to amplify our RT products for CSF analysis. All methods were sensitive and accurate, and each was repeatable and precise.

\section{Analysis of individual miRNAs with TaqMan hydrolysis probes}

Individual TaqMan hydrolysis probes (Applied Biosystems) were used to assess circulating miRNA candidates in the validation samples. The expression of each gene was determined by real-time PCR on a Light-Cycler ${ }^{\circledR} 480$ real-time PCR instrument from Roche Diagnostics (Germany). The most stable miRNA was selected to normalize expression levels (miRNA-17).

\section{Measurement of inflammatory cytokines}

Inflammatory cytokines, IL $-1 \beta$, IL -6, and TNF-a, CSF levels were determined by ELISA according to the instructions of Elabscience Biotechnology Inc. (Cat. No. E-EL-H0149, China), Boster Biological Technology Inc. (Cat. NO. EK0410, USA) and Abcam (Cat. NO. ab181421, USA) kits, respectively. The level of hs-CRP value was determined by the quantitative turbidometric method

\section{Statistical Analysis}

Frequency and percentage were used to represent qualitative data, whereas the mean and standard deviation was used to describe quantitative data. The OneSample Kolmogorov-Smirnov Test was used to examine the normal distribution of the quantitative data. Because the age distribution between the case and control groups was normal, the t-test was used to compare the mean age of subjects between the case and control groups. Because of the abnormal distribution of data related to the biomarkers of microRNAs and inflammatory factors in CSF, the Mann-Whitney test was used to evaluate the levels of these biomarkers between case and control groups. The Spearman test was used to evaluate the relationship between the expression of microRNAs and inflammatory factors in CSF. ROC analysis was also used to evaluate the diagnostic power of microRNA biomarkers in patients with MS. Statistical analysis was performed using SPSS software version 23, and Graph Pad Prism version 8 was used to draw graphs.

\section{Results}

\section{Characteristics of the subjects}

Of the 50 samples studied, 25 samples were from patients with MS, and 25 were from the control group. The number of women ( $n=14,56 \%)$ and men ( $n=11$, $44 \%$ ) was equal in both groups. The mean age of the subjects of the case group was $35.8 \pm 4.4$ years, and in the control group, it was $33.4 \pm 0.4$ years, and the observed difference was not significant $(P>0.05)$. The mean age of onset of MS of the case group was $31.8 \pm 3.8$ years (minimum 27 and maximum 40 ) years, and the mean disease duration was $3.9 \pm 1.4$ (minimum 2 and maximum 6 ) years. Of the 25 patients with MS, 17 (68\%) were treated with medication, and $8(32 \%)$ were not treated.

\section{Inflammatory cytokines concentrations in CSF}


As shown in Figure 1, there were significant differences in CSF levels of the inflammatory cytokines IL-1 $\beta$, IL-6, and TNF- $\alpha$ and hs-CRP between the case and control groups.

According to the results of Table 2, the expression of microRNAs and inflammatory factors was higher in the CSF of patients with MS than in the control group, and this difference was significant $(P<0.05)$ (Tables 1, Figure 1).

Table 1

Biochemical characteristics and miRNA expression between case and control

\begin{tabular}{|c|c|c|c|c|c|}
\hline \multirow[t]{2}{*}{ variable } & \multicolumn{2}{|c|}{ Case $(n=25)$} & \multicolumn{2}{|c|}{ Control $(n=25)$} & \multirow[t]{2}{*}{ P-value ${ }^{*}$} \\
\hline & Median & $Q_{1}-Q_{3}$ & Median & $Q_{1}-Q_{3}$ & \\
\hline IL-6(pg/mL) & 13.40 & $12.90-14.30$ & 0.90 & $0.50-1.10$ & $<0.0001$ \\
\hline $\mathrm{IL}-1 \beta(\mathrm{Pg} / \mathrm{mL})$ & 72.20 & $71.10-74.95$ & 5.50 & $4.55-6.20$ & $<0.0001$ \\
\hline TNF-a (pg/mL) & 4.60 & $4.45-4.90$ & 1.90 & $1.60-2.15$ & $<0.0001$ \\
\hline hs-CRP (mg/L) & 5.90 & $5.50-6.25$ & 1.70 & $1.60-1.90$ & $<0.0001$ \\
\hline miRNA-21 & 0.05 & $0.04-0.06$ & 0.02 & $0.01-0.03$ & $<0.0001$ \\
\hline miRNA-155 & 0.05 & $0.04-0.06$ & 0.02 & $0.01-0.03$ & $<0.0001$ \\
\hline miRNA-182 & 0.06 & $0.05-0.07$ & 0.03 & $0.02-0.35$ & $<0.0001$ \\
\hline miRNA-437 & 0.05 & $0.04-0.06$ & 0.04 & $0.03-0.06$ & 0.047 \\
\hline
\end{tabular}

\section{ROC curve Analysis}

The ability of microRNAs to diagnose multiple sclerosis was evaluated using ROC analysis. The results show that the highest area under the curve was obtained for miRNA-21 (AUC $=0.97, \mathrm{P}<0.0001$ ), miRNA-182 (AUC $=0.97, \mathrm{P}<0.0001$ ), and miRNA-155 (AUC $=0.96, \mathrm{P}<0.0001$ ), respectively. Based on the high values under the curve, it can be concluded that these biomarkers have excellent performance in distinguishing people with MS from people without MS. The highest rate of correct diagnoses of MS compared with incorrect diagnoses (LR $+=17$ ) was associated with miRNA-155 at values above 0.045 in CSF (Table 2 and Figure 2).

Table 2

Predictive potential of selected miRNAs as biomarkers for patients with MS

\begin{tabular}{|c|c|c|c|c|c|c|c|c|c|}
\hline AUC & SE & $95 \% \mathrm{Cl}$ & P-value & Cut off value & Sensitivity & Specificity & $\mathrm{LR}^{+}$ & LR- & \\
\hline miRNA-21 & 0.97 & 0.02 & 0.92 to 1.00 & $<0.0001$ & $>0.035$ & 0.92 & 0.88 & 7.67 & 0.090 \\
\hline miRNA-155 & 0.96 & 0.02 & 0.91 to 1.0 & $<0.0001$ & $>0.045$ & 0.68 & 0.96 & 17 & 0.045 \\
\hline miRNA-182 & 0.97 & 0.02 & 0.93 to 1.0 & $<0.0001$ & $>0.045$ & 0.88 & 0.92 & 11 & 0.13 \\
\hline miRNA-437 & 0.66 & 0.08 & 0.51 to 0.81 & 0.0535 & $>0.065$ & 0.80 & 0.96 & 2 & 0.2 \\
\hline
\end{tabular}

\section{Differentially expressed miRNAs in MS patients and control groups}

As shown in Figure 3, the statistical significance of the groups was evaluated based on the altered expression of 4 miRNAs found in the CSF samples. The groups showed a significant increase in the expression of miRNA-21, miRNA-155, miRNA-182, and miRNA-437.

\section{Correlation between miRNAs and inflammatory cytokines in cerebrospinal fluid.}

When the relationship between the levels of inflammatory cytokines and microRNAs in the CSF of the entire population studied (case and control combined, $n$ $=50$ ) was examined, the results showed that the relationship between inflammatory cytokines and miRNA-21, miRNA-155, and miRNA-182 was statistically significant (Figures 4, 5, 6, and 7), but between miRNA-182 and TNF-a between case and control patients $(r=0.42, P=0.04)$, there was a direct and moderate correlation. There was an indirect and moderate correlation between miRNA-437 and hs-CRP $(r=-0.47, P=0.02)$, which were also statistically significant (Table 3 and Figure 8).

Table 3. The correlation between expression levels of miRNAs and inflammatory factors of IL6, IL-1 $\beta$, TNF- $a$, and hs-CRP in the CSF of patients with MS and control groups. 


\begin{tabular}{|c|c|c|c|c|c|c|c|c|c|c|c|c|c|c|c|c|}
\hline & \multicolumn{4}{|c|}{ miRNA-21 } & \multicolumn{4}{|c|}{ miRNA-155 } & \multicolumn{6}{|c|}{ miRNA-182 } & \multirow{2}{*}{\multicolumn{2}{|c|}{$\begin{array}{l}\text { miRNA-437 } \\
\text { Case }\end{array}$}} \\
\hline & Contrc & & Case & & Contro & & Case & & Contrc & & Case & & Contro & & & \\
\hline & $\mathbf{r}$ & $\begin{array}{l}\mathrm{P} \\
\text { value }^{*}\end{array}$ & $r$ & $\begin{array}{l}P \\
\text { value }^{*}\end{array}$ & $\mathbf{r}$ & $\begin{array}{l}P \\
\text { value }^{*}\end{array}$ & $r$ & $\begin{array}{l}P \\
\text { value }^{*}\end{array}$ & r & $\begin{array}{l}P \\
\text { value }^{*}\end{array}$ & $r$ & $\begin{array}{l}P \\
\text { value }^{*}\end{array}$ & $r$ & $\begin{array}{l}\mathrm{P} \\
\text { value }^{*}\end{array}$ & $r$ & $\begin{array}{l}\mathrm{P} \\
\text { value }\end{array}$ \\
\hline $\mathrm{IL}-6(\mathrm{pg} / \mathrm{mL})$ & 0.01 & 0.96 & 0.20 & 0.33 & -0.25 & 0.23 & -0.18 & 0.38 & 0.35 & 0.09 & -0.17 & 0.42 & -0.02 & 0.90 & -0.05 & 0.83 \\
\hline $\mathrm{IL}-1 \beta(\mathrm{Pg} / \mathrm{mL})$ & 0.26 & 0.21 & -0.07 & 0.75 & -0.12 & 0.56 & 0.07 & 0.72 & -0.01 & 0.99 & 0.22 & 0.28 & -0.06 & 0.77 & -0.02 & 0.91 \\
\hline $\begin{array}{l}\text { TNF-a } \\
(\mathrm{pg} / \mathrm{mL})\end{array}$ & 0.18 & 0.37 & -0.06 & 0.79 & -0.47 & 0.02 & -0.21 & 0.32 & 0.04 & 0.83 & -0.47 & 0.02 & -0.36 & 0.08 & -0.12 & 0.57 \\
\hline $\begin{array}{l}\text { hs- } \\
\text { CRP(mg/L) }\end{array}$ & -0.45 & 0.02 & -0.26 & 0.21 & 0.27 & 0.19 & 0.08 & 0.71 & 0.03 & 0.90 & -0.08 & 0.69 & -0.02 & 0.94 & 0.42 & 0.04 \\
\hline
\end{tabular}

*Spearman test was used.

\section{Discussion}

We evaluated the effect of miRNAs on inflammatory factors, including IL-1 $\beta$, IL-6, TNF-a, and hs-CRP, in developing MS. Analysis of miRNA dysfunction and changes in inflammatory factors can help better understand the cause of MS and new alternative methods of diagnosing and treating the disease. This study found that the association between IL-1 $\beta$, IL-6, TNF- $\alpha$, and hs-CRP and miRNAs that are involved in developing MS. We selected several miRNAs to investigate their association with inflammatory factors: miRNA-21, miRNA-155, miRNA-182, and miRNA-437. We examined the levels of IL-1 3 , IL-6, TNF-a, hs-CRP, and miRNAs in the cerebrospinal fluid of MS patients and healthy individuals.

Studies have examined the expression of miRNAs in cell-free cerebrospinal fluid [28-30], a biological fluid reflecting events in the central nervous system. However, no study has examined the association with active multiple sclerosis lesions. The differential miRNA expression in CSF from MS patients is a valuable indication of CNS inflammation. One study discovered miRNAs in the CSF and serum of dogs having neurological disorders. It was found that microRNA-21 levels in the CSF of dogs with MUO were higher than in dogs with OND and normal CSF NCC [31]. Fenoglio et al. discovered that miRNA-21 was overrepresented in the PBMCs of patients with recurrent MS compared to control subjects. They hypothesized that this increase occurs entirely in the acute phase of MS and provides to producing and controlling CD4+ T cells involved in the inflammatory processes affecting the central nervous system associated with MS [32,33]. miRNA-21 is involved in T cell activation and death, Treg cell activity and growth, and Th17 cell differentiation [34]. miRNA-155 is an essential regulator of inflammation and contributes to the modulation of the autoimmune response in MS. miRNA-155 is involved in Blood-Brain Barrier (BBB) breakdown under inflammatory conditions by downregulating critical junctional proteins [35]. Additionally, one study found that MS patients had higher expression of miRNA-182-5p than the control group [36]. Another study found that individuals with depression had increased miRNA-182-5p expression in blood [37]. These results confirm our observations.

Changes in miRNAs in CSF reflect changes in miRNAs in diseased brain tissue more accurately than changes in blood or postmortem brain samples [38]. This event can be explained by one of 2 different mechanisms: (1) Due to the inherent difficulties in sampling brain tissue in vivo, miRNA expression in human research is predominantly studied using postmortem samples. However, prolonged postmortem delay and previous drug use affect the study of postmortem brain tissue;(2) Peripheral blood cannot accurately represent the gene expression level in the brain. Because the brain is closely related to the CSF, CSF markers allow the discovery of new CNS biomarkers. Wan et al. identified several miRNAs in cerebrospinal fluid (CSF) from patients with MDD [39, 40]. Results showed that the relationship between inflammatory cytokines and miRNA-21, miRNA-155, and miRNA-182 was statistically significant. Still, between miRNA-182 and TNF-a between case and control patients, there was a direct and moderate correlation. There was an indirect and moderate correlation between miRNA-437 and hs-CRP, which were also statistically significant. MiRNA-155 is an essential regulator of inflammation and contributes to the modulation of the autoimmune response in MS. Emerging evidence suggests that neuropathic pain is a neuro-immune dysfunction associated with increased immune system activity. miRNA-155 has a well-characterized effect on inflammatory variables associated with neuropathic pain, including interleukin-1, interleukin-6, TNF-, NF$B$, and p38 mitogen-activated protein kinase (MAPK) [41-43]. The miRNA-155 expression has been increased in the prefrontal cortex of rats suffering from inflammatory pain [44]. MicroRNAs affect the differentiation and function of inflammatory cells. MiRNA-182 is a transcription factor that regulates FOXO1 expression in T cells and influences cell differentiation and activation. Additionally, miRNA-21 promotes T cell progression to Th1, IFN production, and Foxp3 expression. In astrocyte cells, miRNA-155 controls the production of pro-inflammatory genes [24]. In our study, an increase in miRNA-155 was associated with an increase in inflammatory variables. Further results revealed a statistical relationship between pro-inflammatory cytokines and miRNA-182 and miRNA-437. Our results suggest that these microRNAs could be used as therapeutic targets in the future.

Many studies have shown that miRNA-21 plays a critical role in terminating inflammation and negatively limits the pro-inflammatory response elicited by many of the same triggers that increase miRNA-21. In macrophages, miRNA-21 is a critical modulator of the anti-inflammatory response [45]. In individuals with Gd+ MS, a steady increase in miRNA-21 was observed over time. There was a positive correlation between this increase and the frequency of Gd+ lesions and neurofilament light chain (NF-L) concentration [46]. However, Yang et al [47]. demonstrated that mice lacking miRNA-21 had worse survival rates, more severe cardiac dysfunction, and more extensive infarct and scar regions after myocardial infarction) (MI) compared with WT mice. MiRNA-21 knockout mice had higher levels of pro-inflammatory cytokines such as IL-1, IL-6, and TNF-in cardiac tissue and increased infiltration of CD11b+ monocytes/macrophages with pro-inflammatory cytokine production. In our study, miRNA-21 expression was increased in the CSF of MS patients. This increase in expression increased 
the level of inflammatory factors. Comparison of the results of our study with other studies showed that changes in miRNA-21 expression are different in different tissues and diseases.

The results of ROC analysis show that the highest area under the curve was obtained for miRNA-21 (97), miRNA-182 (97), miRNA-155 (96), and miRNA437(66). Based on the high values under the curve, it can be concluded that biomarkers have excellent performance in distinguishing people with MS from people without MS. They can be used to follow the healing process [48]. Which miRNAs were associated with the highest rate of correct MS diagnoses compared with misdiagnoses? MiRNA-155 in CSF was associated with the highest percentage of accurate MS diagnoses compared with misdiagnoses.

\section{Conclusion}

Patients with multiple sclerosis have increased expression of miRNAs such as miRNA-21, miRNA-155, miRNA-182, and miRNA-437 and inflammatory markers in cerebrospinal fluid (CSF). Increased expression of miRNA-21, miRNA-155, miRNA-182, and miRNA-437 in CSF decreases neuroprotective activity, which may be due to the immune system switching to inflammation through the production of IL -1, IL -6, TNF-, and hs-CRP. An increase in some miRNAs, including miRNA-21, miRNA-155, miRNA-182, and miRNA-437, may contribute to the maintenance of chronic inflammation in MS. Our results suggest that the levels of $\mathrm{IL}-1 \beta, \mathrm{IL}-6, \mathrm{TNF}-\mathrm{a}$, and hs-CRP, and selected miRNAs in CSF, serve as biomarkers of CNS inflammation and neurodegenerative processes in patients with multiple sclerosis.

\section{Declarations}

\section{Acknowledgments}

The authors thank Tabriz University of Medical Sciences for their financial support. We also thank the staff of the Neurosciences Research Center for their cooperation and advice.

\section{Contribution of Author}

VK, FS, MH, DL, SA and BSH performed all analyses and experiments. AR and MN supervised the experiments. AN supervised the study and confirmed the draft manuscript

\section{Availability of data and materials}

The data and materials used in this study are available.

\section{Conflict of interest}

The authors declare that there are no conflicts of interest

\section{Ethical approval}

The Ethics Committee of the Tabriz University of medical sciences approved the study protocols following the Declaration of Helsinki.

\section{Informed consent}

the informed consent of all patients in this study has been obtained. The Ethics Committee of Tabriz University of Medical Sciences approved this study's protocol, and it was carried out following the Helsinki Declaration of the World Medical Association.

\section{Consent for publication}

Not applicable.

\section{References}

1. Bolton C, Smith PA. Defining and regulating acute inflammatory lesion formation during the pathogenesis of multiple sclerosis and experimental autoimmune encephalomyelitis. CNS Neurol Disord Drug Targets. 2015;14: 915-35.

2. Shademan B, Biray Avci C, Nikanfar M, Nourazarian A. Application of Next-Generation Sequencing in Neurodegenerative Diseases: Opportunities and Challenges. Neuromolecular medicine. 2021 Jun;23(2):225-35.

3. Dutta R, Chang A, Doud MK, Kidd GJ, Ribaudo MV, Young EA, Fox RJ, Staugaitis SM, Trapp BD. Demyelination causes synaptic alterations in hippocampi from multiple sclerosis patients. Annals of neurology. 2011 Mar;69(3):445-54.

4. Alam A, Thelin EP, Tajsic T, Khan DZ, Khellaf A, Patani R, Helmy A. Cellular infiltration in traumatic brain injury. Journal of neuroinflammation. 2020 Dec;17(1):1-7.

5. Scazzone C, Agnello L, Sasso BL, Ragonese P, Bivona G, Realmuto S, lacolino G, Gambino CM, Bellia C, Salemi G, Ciaccio M. Klotho and vitamin D in MS: an Italian study. Archives of Medical Science: AMS. 2020;16(4):842. 
6. A, Stojić M, Dežmalj L, Tudorić-Đeno I, Romić D, Jeleč V, Vrca A, Vuletić V, Grčević D. RANKL/RANK/OPG axis is deregulated in the cerebrospinal fluid of multiple sclerosis patients at clinical onset. Neuroimmunomodulation. 2018;25:23-33.

7. Malekzadeh A, de Geer-Peeters V, De Groot V, Elisabeth Teunissen C, Beckerman H. Fatigue in patients with multiple sclerosis: is it related to pro-and antiinflammatory cytokines?. Disease markers. 2015 Oct;2015.

8. Kothur K, Wienholt L, Brilot F, Dale RC. CSF cytokines/chemokines as biomarkers in neuroinflammatory CNS disorders: a systematic review. Cytokine. 2016 Jan 1;77:227-37.

9. Hofer LS, Mariotto S, Wurth S, Ferrari S, Mancinelli CR, Delogu R, Monaco S, Gajofatto A, Schwaiger C, Rostasy K, Deisenhammer F. Distinct serum and cerebrospinal fluid cytokine and chemokine profiles in autoantibody-associated demyelinating diseases. MS Journal-Experimental, Translational and Clinical. 2019 May;5(2):2055217319848463.

10. Matejcikova Z, Mares J, Prikrylova Vranova H, Klosova J, Sladkova V, Dolakova J, et al. Cerebrospinal fluid inflammatory markers in patients with multiple sclerosis: a pilot study. J Neural Transm. (Vienna). 2015; 122:273-7. doi:10.1007/s00702-014-1244-9

11. Mousaki A, Rodi M, Dimisianos N, Emmanuil A, Kalavrizioti D, Lagoudaki R, et al. Immune parameters that distinguish multiple sclerosis patients from patients with other neurological disorders at presentation. PLoS One. 2015; 10:e0135434.

12. Sosvorova L, Kanceva R, Vcelak J, Kancheva L, Mohapl M, Starka L, Havrdova E. The comparison of selected cerebrospinal fluid and serum cytokine levels in patients with multiple sclerosis and normal pressure hydrocephalus. Neuroendocrinol Lett. 2015 Jan 1;36(6):564-71.

13. Peiravian F, Rajaian H, Samiei A, Gholijani N, Gharesi-Fard B, Mokaram P, et al. Altered serum cytokine profiles in relapse phase of relapsing-remitting multiple sclerosis. Iran J Immunol. 2016; 13:186-96.

14. Sproston NR, Ashworth JJ. Role of C-reactive protein at sites of inflammation and infection. Frontiers in immunology. 2018 Apr 13;9:754.

15. Eklund CM. Proinflammatory cytokines in CRP baseline regulation. Advances in clinical chemistry. 2009;48:111-36.

16. Boylan MT, Crockard AD, Duddy ME, Armstrong MA, McMillan SA, Hawkins SA. Interferon- $\beta 1$ a administration results in a transient increase of serum amyloid A protein and C-reactive protein: comparison with other markers of inflammation. Immunology letters. 2001 Jan 15;75(3):191-7.

17. Tehrani A, Gholipour S, Sharifi R, Yadegari S, Abbasi-Kolli M, Masoudian N. Plasma levels of CTRP-3, CTRP-9 and apelin in women with MS. J Neuroimmunol 2019.

18. Jalkanen A, Kauko T, Koskinen JO, Waris ME, Airas L. Elevated concentration of C-reactive protein is associated with pregnancy-related co-morbidities but not with relapse activity in multiple sclerosis. Neurol Sci. 2015; 36:441-7.

19. Ji AL, Liu ZH, Chen WW, Huang WJ. The clinical significance of level changes of hs-CRP, IL-10 and TNF for patients with MS during active and relieving period. Eur Rev Med Pharm Sci. 2016;20:4274-6.

20. Gunel, N.S., Birden, N., Kurt, C.C. et al. Effect of valproic acid on miRNAs affecting histone deacetylase in a model of anaplastic thyroid cancer. Mol Biol Rep.2021:6085-6091.

21. Halushka, M.K.; Fromm, B.; Peterson, K.J.; McCal, M.N. Big strides in cellular microRNA expression. Trends Genet. 2018, 34, $165-167$.

22. Cui C, Cui Q. The relationship of human tissue microRNAs with those from body fluids. Scientific reports. 2020 Mar 27;10(1):1-7.

23. Ceman S, Saugstad J. MicroRNAs: Meta-controllers of gene expression in synaptic activity emerge as genetic and diagnostic markers of human disease. Pharmacology \& therapeutics. 2011 Apr 1;130(1):26-37.

24. Ksiazek-Winiarek DJ, Kacperska MJ, Glabinski A. MicroRNAs as novel regulators of neuroinflammation. Mediators of inflammation. 2013 Oct; 2013.

25. A. Junker, M. Krumbholz, S. Eisele et al., "MicroRNA profiling of multiple sclerosis lesions identifies modulators of the regulatory protein CD47," Brain, vol. 132, no. 12, pp. 3342-3352, 20.

26. Ceppi M, Pereira PM, Dunand-Sauthier I, Barras E, Reith W, Santos MA, Pierre P. MicroRNA-155 modulates the interleukin-1 signaling pathway in activated human monocyte-derived dendritic cells. Proceedings of the national academy of sciences. 2009 Feb 24;106(8):2735-40.

27. Khajenobar NB, Mahboob S, Nourazarian A, Shademan B, Laghousi D, Moayed ZB, Hassanpour M, Nikanfar M. Comparison between cerebrospinal fluid and serum levels of myelin-associated glycoprotein, total antioxidant capacity, and 8-hydroxy-2'-deoxyguanosine in patients with multiple sclerosis. Clinical Neurology and Neurosurgery. 2021 Jan 1;200:106377.

28. Kopkova A, Sana J, Fadrus P, Slaby O. Cerebrospinal fluid microRNAs as diagnostic biomarkers in brain tumors. Clinical Chemistry and Laboratory Medicine (CCLM). 2018 Jun 1;56(6):869-79.

29. Wu KQ, Zhang SF, Bao CH, Zou X, Gu X, Wang CN, Gong WM, Shi M, Lou YL, Huang J, Zhou PY. Circulating MicroRNAs as Potential Biomarkers in the Diagnosis of Neurosyphilis: A Case Control Study. International Journal of Dermatology and Venereology. 2021 Mar 1;4(1):16-25.

Page 7/13 
30. Perdaens O, Dang HA, D'Auria L, Van Pesch V. CSF microRNAs discriminate MS activity and share similarity to other neuroinflammatory disorders. Neurology-Neuroimmunology Neuroinflammation. 2020 Mar 5;7(2).

31. Gaitero L, Russell SJ, Monteith G, LaMarre J. Expression of microRNAs miR-21 and miR-181c in cerebrospinal fluid and serum in canine meningoencephalomyelitis of unknown origin. The Veterinary Journal. 2016 Oct 1;216:122-4.

32. Fenoglio C, Cantoni C, De Riz M, Ridolfi E, Cortini F, Serpente M, Villa C, Comi C, Monaco F, Mellesi L, Valzelli S. Expression and genetic Analysis of miRNAs involved in CD4+ cell activation in patients with multiple sclerosis. Neuroscience letters. 2011 Oct 17;504(1):9-12.

33. Sospedra M, Martin R. Immunology of multiple sclerosis. Annu. Rev. Immunol.. 2005 Apr 23;23:683-747.

34. Wang S, Wan X, Ruan Q. The microRNA-21 in autoimmune diseases. International journal of molecular sciences. 2016 Jun;17(6):864.

35. Maciak K, Dziedzic A, Miller E, Saluk-Bijak J. miR-155 as an Important Regulator of Multiple Sclerosis Pathogenesis. A Review. International Journal of Molecular Sciences. 2021 Jan;22(9):4332.

36. Piotrzkowska D, Miller E, Kucharska E, Niwald M, Majsterek I. Association of miRNA and mRNA Levels of the Clinical Onset of Multiple Sclerosis Patients. Biology. 2021 Jun;10(6):554.

37. Li Y.J., Xu M., Gao Z.H., Wang Y.Q., Yue Z., Zhang Y.X., Li X.X., Zhang C., Xie S.Y., Wang P.Y. Alterations of Serum Levels of BDNF-Related miRNAs in Patients with Depression. PLoS ONE. 2013;8:e63648.

38. Gallego JA, Gordon ML, Claycomb K, Bhatt M, Lencz T, Malhotra AK. In vivo microRNA detection and quantitation in cerebrospinal fluid. Journal of Molecular Neuroscience. 2012 Jun;47(2):243-8.

39. McKeever PM, Schneider R, Taghdiri F, Weichert A, Multani N, Brown RA, Boxer AL, Karydas A, Miller B, Robertson J, Tartaglia MC. MicroRNA expression levels are altered in the cerebrospinal fluid of patients with young-onset Alzheimer's disease. Molecular neurobiology. 2018 Dec;55(12):8826-41.

40. Akers JC, Hua W, Li H, Ramakrishnan V, Yang Z, Quan K, Zhu W, Li J, Figueroa J, Hirshman BR, Miller B. A cerebrospinal fluid microRNA signature as biomarker for glioblastoma. Oncotarget. 2017 Sep 15;8(40):68769.

41. Vallejo R, Tilley DM, Vogel L, Benyamin R. The role of glia and the immune system in the development and maintenance of neuropathic pain. Pain practice. 2010 May;10(3):167-84.

42. Ledeboer, A.; Gamanos, M.; Lai, W.; Martín, D.; Maier, S.F.; Watkins, L.R.; Quan, N. Involvement of spinal cord nuclear factor KB activation in rat models of pro-inflammatory cytokine-mediated pain facilitation. Eur. J. Neurosci. 2005, 22, 1977-1986.

43. Xu, L.; Huang, Y.; Yu, X.; Yue, J.; Yang, N.; Zuo, P. The Influence of p38 Mitogen-Activated Protein Kinase Inhibitor on Synthesis of Inflammatory Cytokine Tumor Necrosis Factor Alpha in Spinal Cord of Rats with Chronic Constriction Injury. Anesthesia Analg. 2007, 105, 1838-1844.

44. Pohl, K.-W.; Yeol, J.-F.; Ongl, W.-Y. MicroRNA changes in the mouse prefrontal cortex after inflammatory pain. Eur. J. Pain 2011, 15, 801.e1-801.e12.

45. Sheedy FJ. Turning 21: induction of miR-21 as a key switch in the inflammatory response. Frontiers in immunology. 2015 Jan $29 ; 6: 19$.

46. Muñoz-San Martín M, Reverter G, Robles-Cedeño R, Buxò M, Ortega FJ, Gómez I, Tomàs-Roig J, Celarain N, Villar LM, Perkal H, Fernández-Real JM. Analysis of miRNA signatures in CSF identifies upregulation of miR-21 and miR-146a/b in patients with multiple sclerosis and active lesions. Journal of neuroinflammation. 2019 Dec;16(1):1-0.

47. Yang L, Wang B, Zhou Q, Wang Y, Liu X, Liu Z, Zhan Z. MicroRNA-21 prevents excessive inflammation and cardiac dysfunction after myocardial infarction through targeting KBTBD7. Cell death \& disease. 2018 Jul 10;9(7):1-4.

48. Shademan B, Nourazarian A, Nikanfar M, Avci CB, Hasanpour M, Isazadeh A. Investigation of the miRNA146a and miRNA155 gene expression levels in patients with multiple sclerosis. Journal of Clinical Neuroscience. 2020 Aug 1;78:189-93.

\section{Figures}



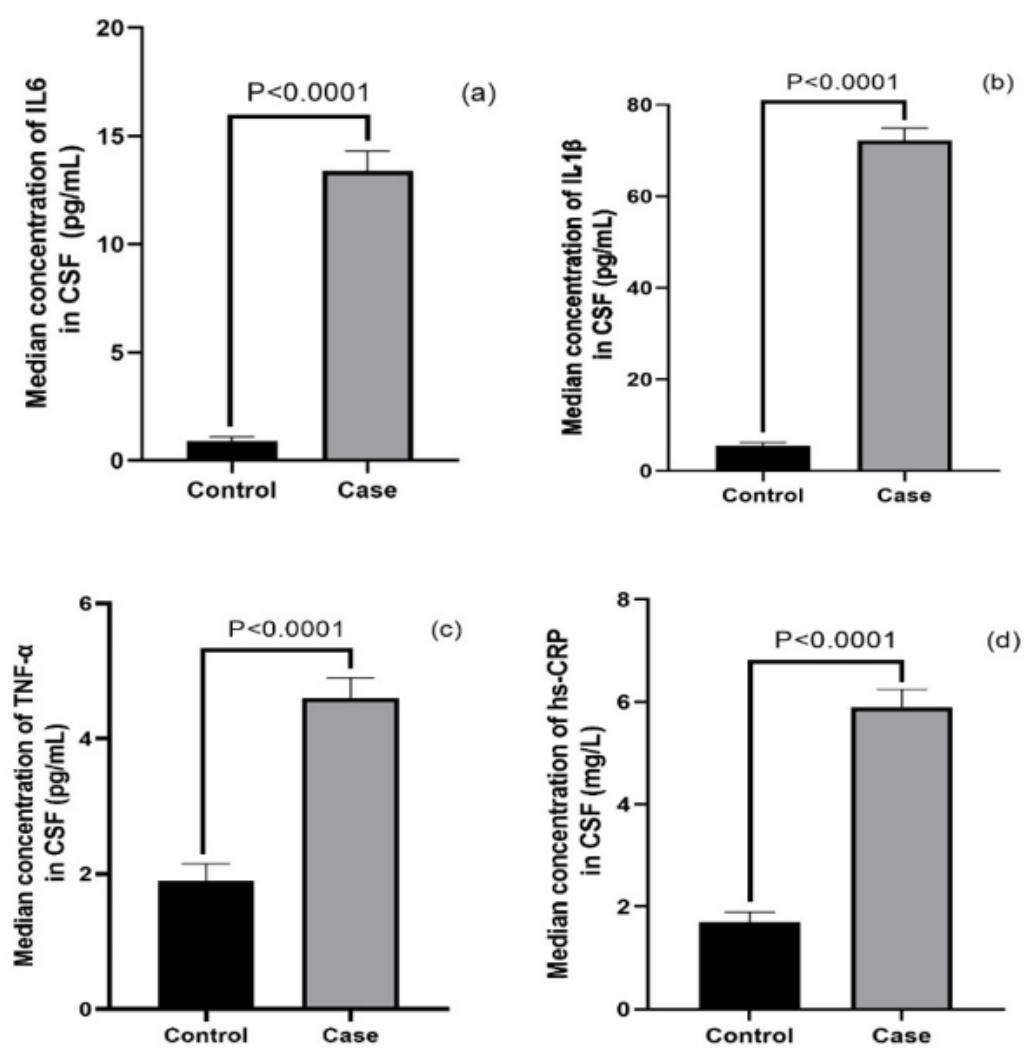

Figure 1

Median and interquartile range (IQR: Q1-Q3) of CSF concentrations of IL6 (a), IL-1ß (b), TNF-a (c), and hs-CRP (d) between case (patients with MS) and control groups. 
(a)
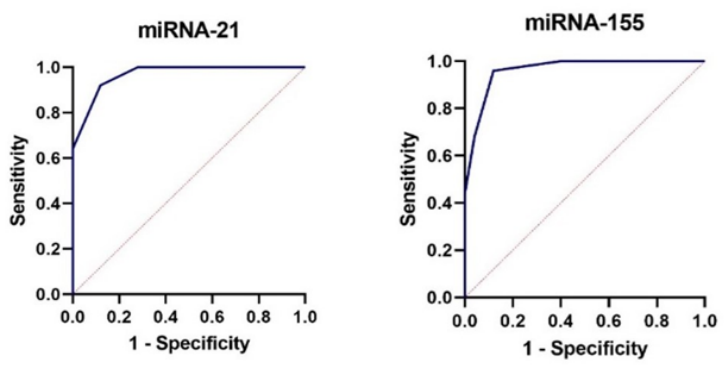

(d)
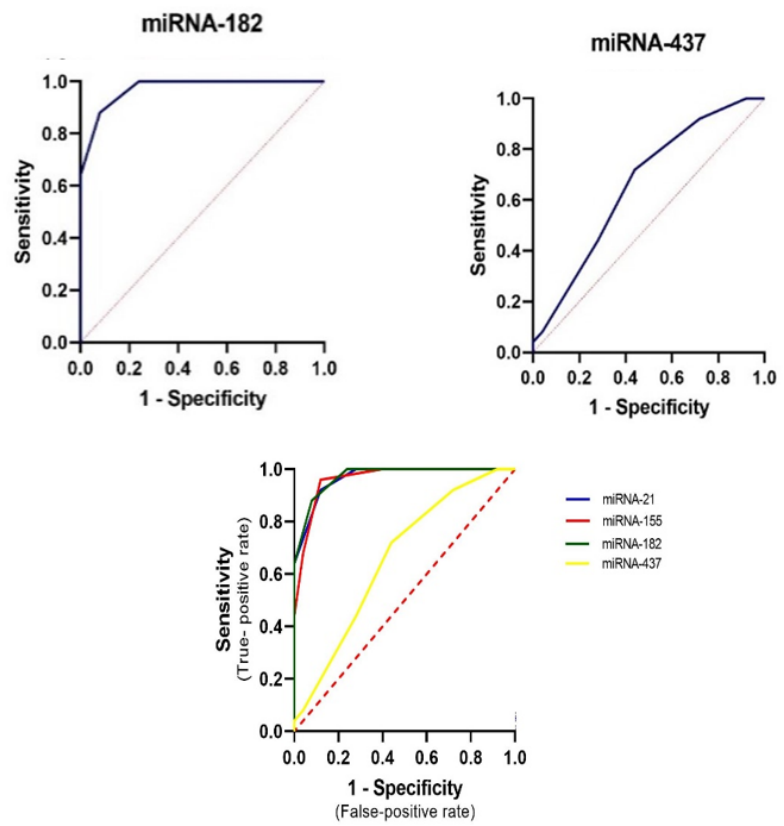

Figure 2

ROC curve analysis for miRNA-21 (a), miRNA-155 (b), miRNA-182 (c), miRNA-437 in MS patients. 


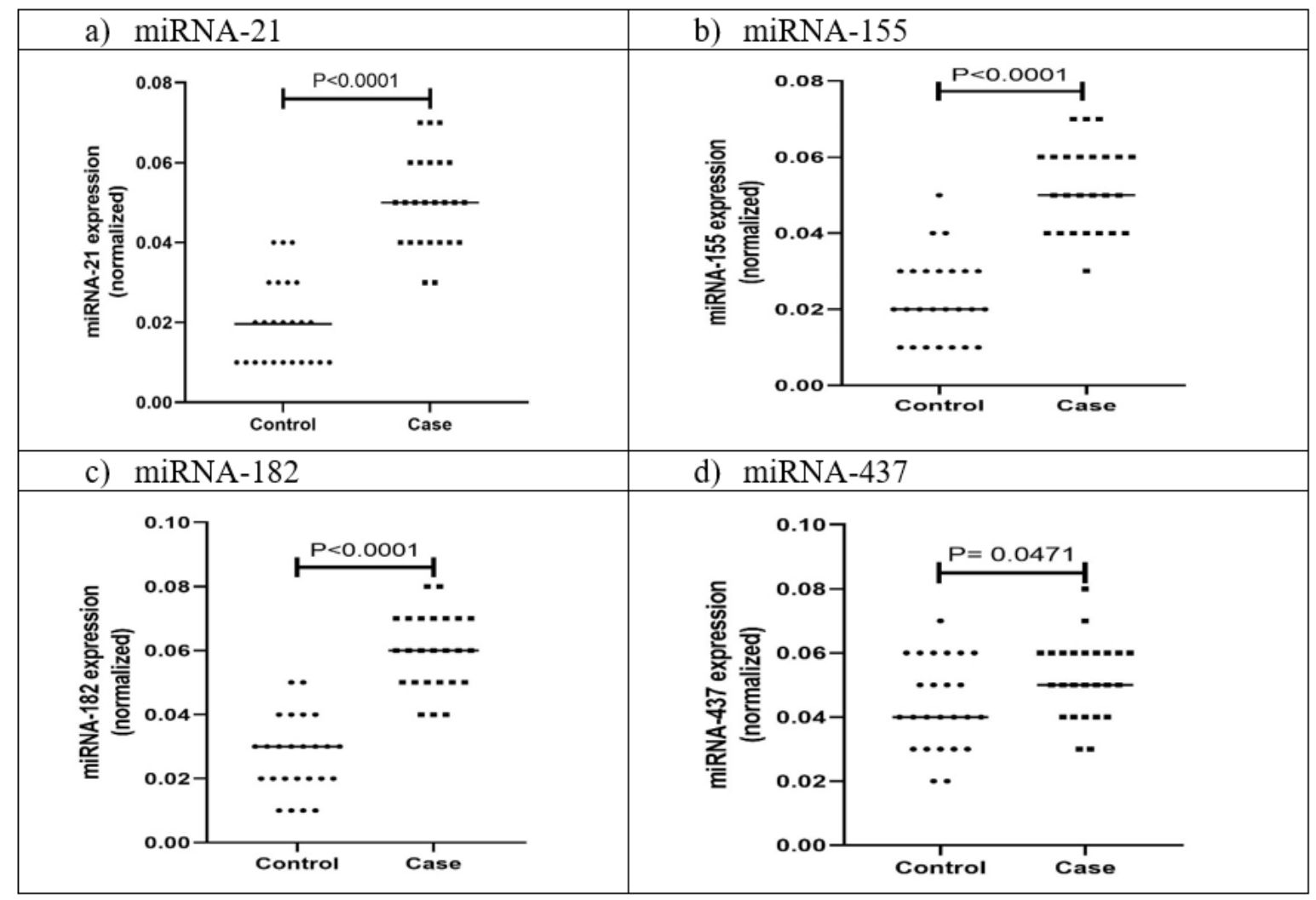

Figure 3

Differentially expressed miRNAs. Dot plots for the normalized value of miRNA-21 (a), miRNA-155 (b), miRNA-182 (c), and miRNA-437 in the case (MS patients) and control groups. The line indicates the median, and the Mann-Whitney $\mathrm{U}$ test was used to determine statistical differences between groups.
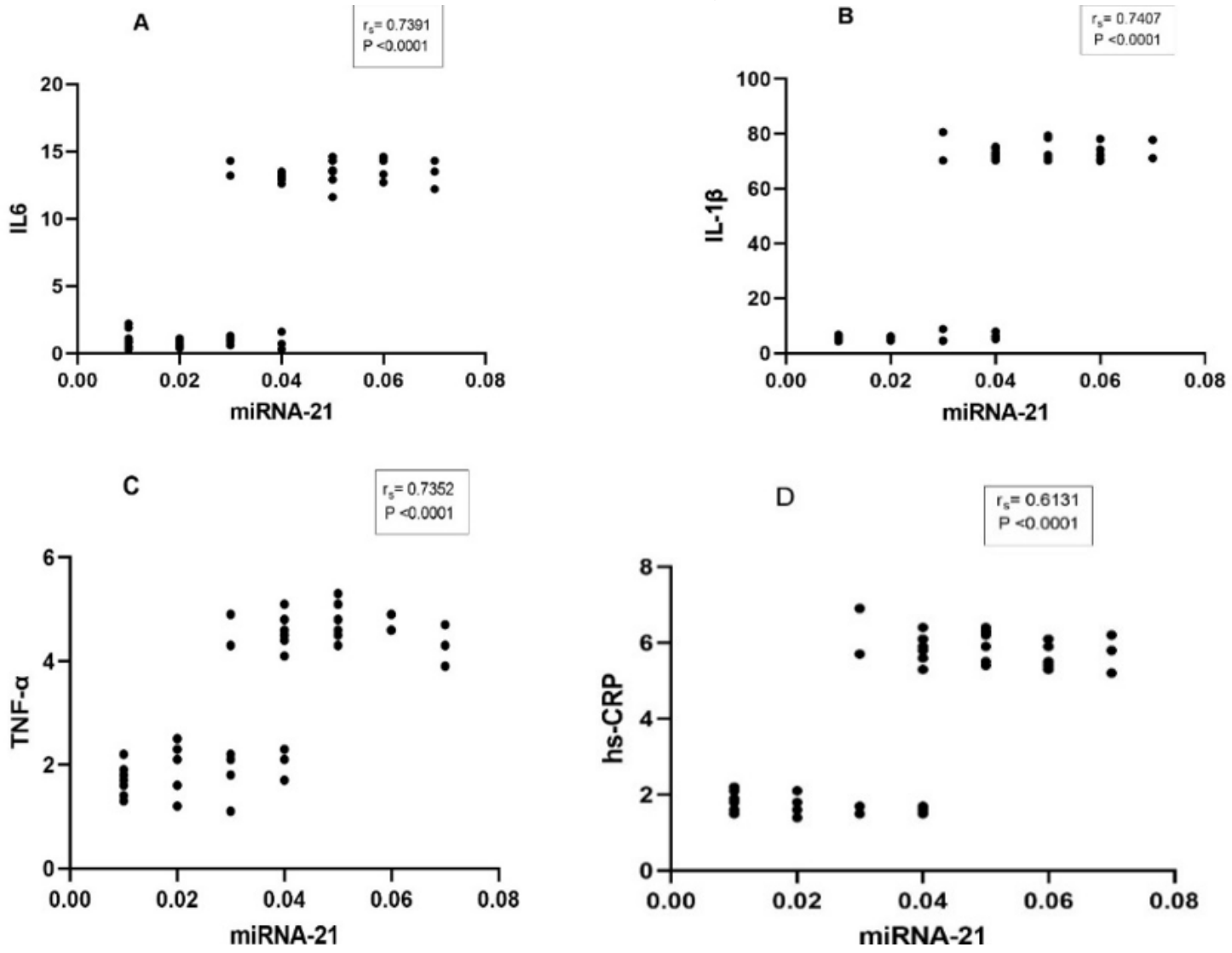

Figure 4 
The correlation between the expression levels of miRNA-21 and the inflammatory factors IL6 (A), IL-1 $\beta$ (B), TNF- $a$ (C), and hs-CRP (D) in CSF. rs=. Rho Spearman
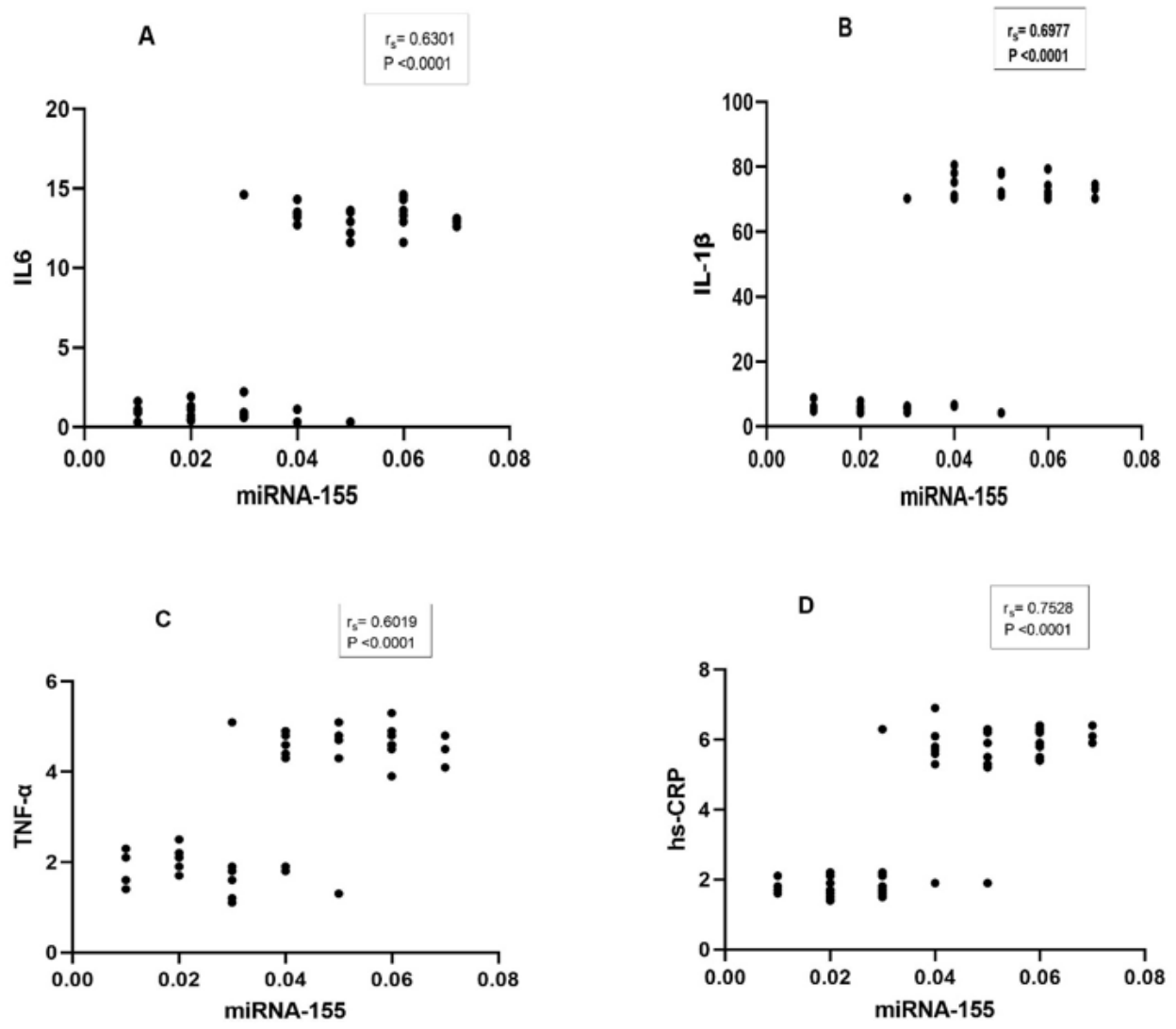

\section{Figure 5}

The correlation between the expression levels of miRNA-155 and the inflammatory factors IL6 (A), IL-1 $\beta$ (B), TNF- $\alpha(C)$, and hs-CRP (D) in CSF. rs=. Rho Spearman
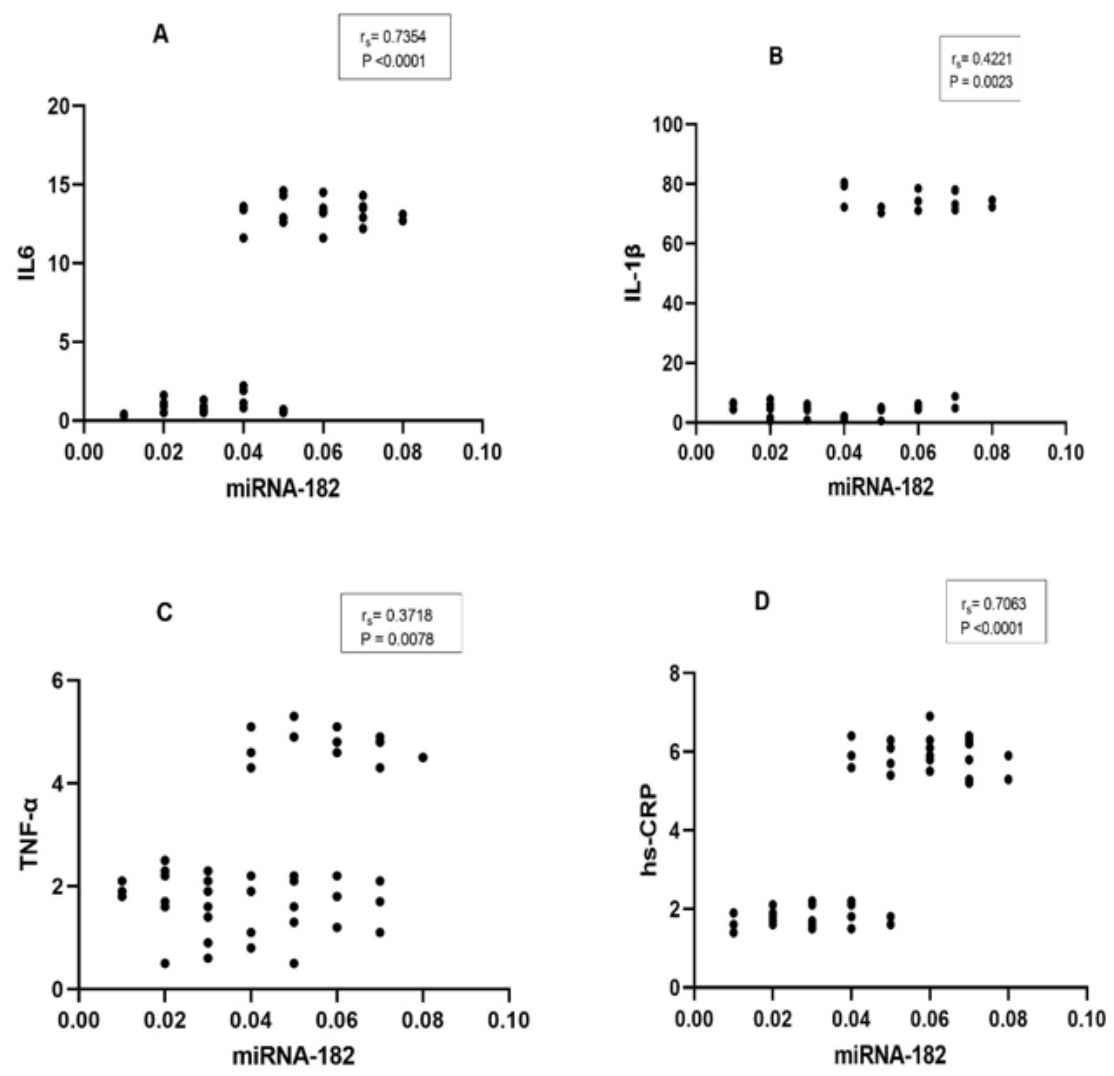

Figure 6

Page 12/13 
correlation between the expression levels of miRNA-182 and the inflammatory factors IL6 (A), IL-1ß (B), TNF- $a$ (C), and hs-CRP (D) in CSF. rs=. Rho Spearman
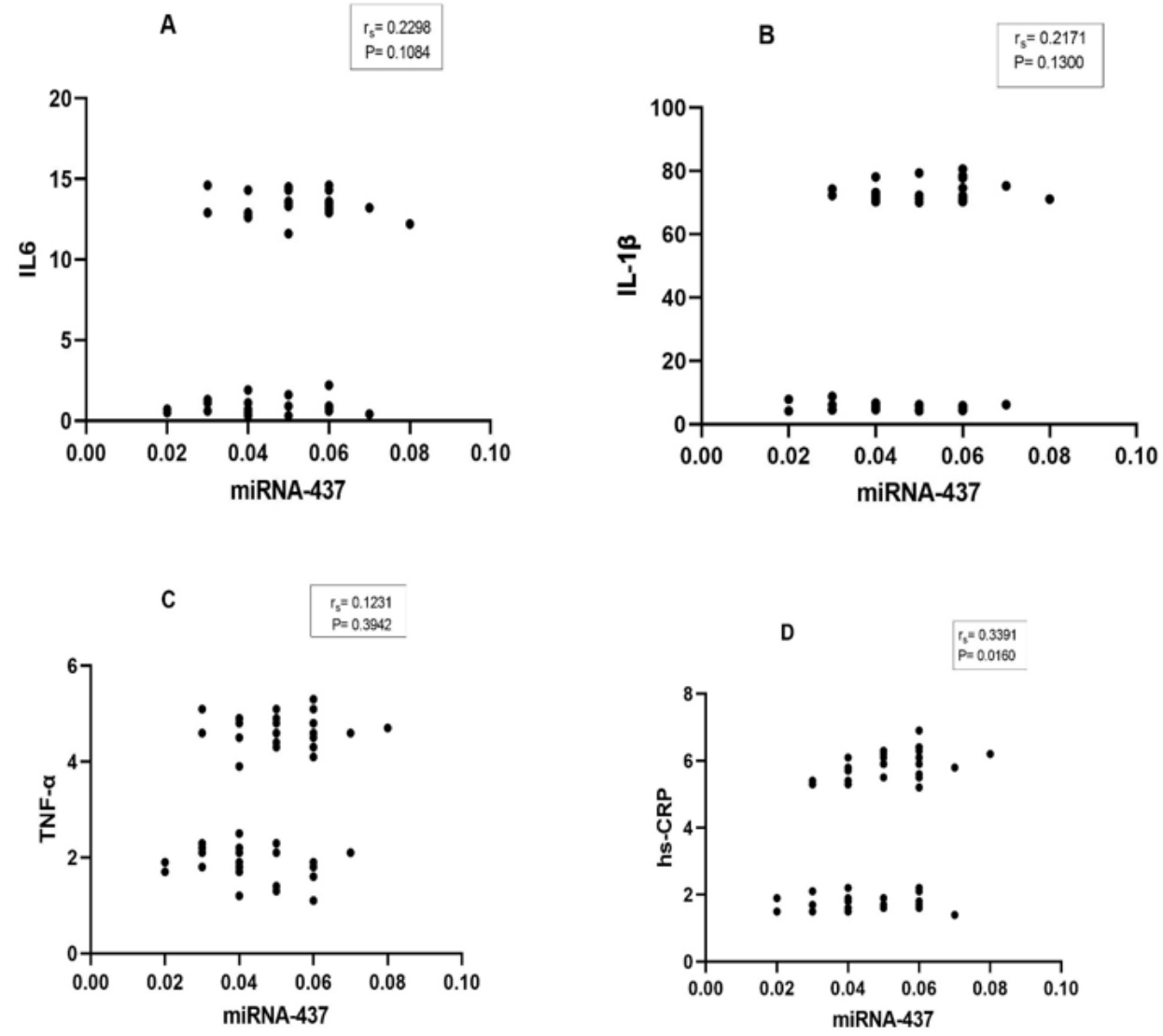

Figure 7

The correlation between the expression levels of miRNA-437 and the inflammatory factors IL6 (A), IL -1 $\beta$ (B), TNF-a (C), and hs-CRP (D) in CSF. rs=. Rho Spearman
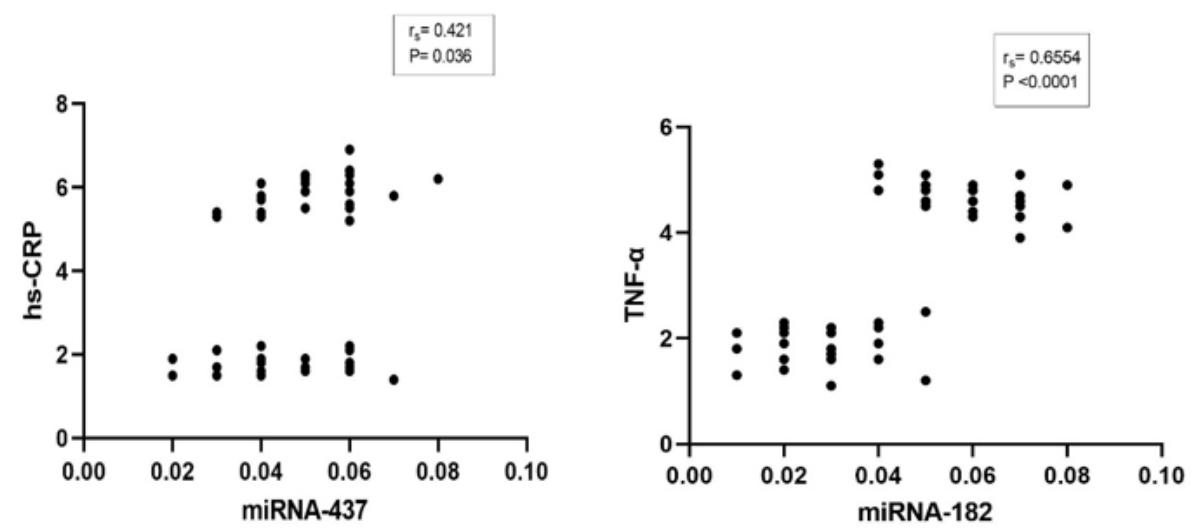

Figure 8

significant correlation between the expression levels of miRNA-437 and miRNA-182 with the inflammatory cytokines TNF-a and hs-CRP in the CSF of patients with MS. rs= Rho Spearman 